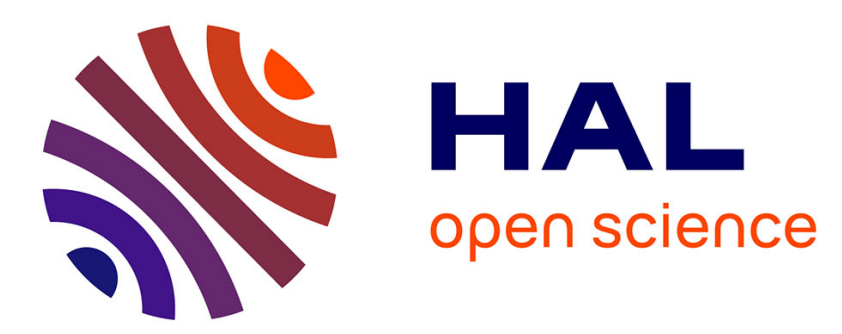

\title{
Résistance au développement d'Heterodera avenae Woll. chez différentes espèces de Triticum
}

Roger Rivoal, Gérard Doussinault, Joseph Jahier, Paulette Penard

\section{To cite this version:}

Roger Rivoal, Gérard Doussinault, Joseph Jahier, Paulette Penard. Résistance au développement d'Heterodera avenae Woll. chez différentes espèces de Triticum. Agronomie, 1986, 6 (8), pp.759-765. hal-00884934

\section{HAL Id: hal-00884934 \\ https://hal.science/hal-00884934}

Submitted on 1 Jan 1986

HAL is a multi-disciplinary open access archive for the deposit and dissemination of scientific research documents, whether they are published or not. The documents may come from teaching and research institutions in France or abroad, or from public or private research centers.
L'archive ouverte pluridisciplinaire HAL, est destinée au dépôt et à la diffusion de documents scientifiques de niveau recherche, publiés ou non, émanant des établissements d'enseignement et de recherche français ou étrangers, des laboratoires publics ou privés. 


\title{
Résistance au développement d'Heterodera ave- nae Woll. chez différentes espèces de Triticum
}

\author{
Roger RIVOAL, Gérard DOUSSINAULT $\left({ }^{*}\right)$ \& Joseph JAHIER (*) \\ avec la collaboration technique de Paulette PENARD \\ I.N.R.A., Laboratoire de Zoologie \\ (*) Station d'Amélioration des Plantes, Centre de Recherches de Rennes, F35650 Le Rheu
}

RÉSUMÉ

\begin{abstract}
Le comportement vis-à-vis de 2 pathotypes d'Heterodera avenae de 88 lignées ou hybrides appartenant aux espèces Triticum monococcum, T. urartu, T. turgidum, T. timopheevi, T. aestivum, T. umbellulatum, T. variabile, a été étudié. Une grande variation est observée dans le caractère hôte des lignées. Des niveaux de résistance très élevés sont mis en évidence chez $T$. monococcum ssp. monococcum, $T$. turgidum ssp. turgidum, $T$. timopheevi ssp. araraticum, T. aestivum ssp. vulgare, $T$. umbellulatum et $T$. variabile. L'utilisation de ces sources de résistance est envisagée pour l'amélioration du blé tendre. Certaines lignées de blés diploïdes et tétraploïdes pourraient constituer des hôtes sélectifs supplémentaires pour la caractérisation des 2 pathotypes d' $H$. avenae utilisés.
\end{abstract}

Mots clés additionnels : Nématode, pathotype, blé, sélection pour la résistance, hôte différentiel.

The behaviour towards 2 pathotypes of Heterodera avenae of 88 lines or hybrids of Triticum monococcum, T. urartu, T. turgidum, T. timopheevi, T. aestivum, T. umbellulatum, and T. variabile was studied on naturally or artificially infested soil. Wide variation was observed in the "host quality" of the lines. Very high levels of resistance were shown in $T$. monococcum ssp. monococcum, T. turgidum ssp. turgidum, T. timopheevi ssp. araraticum, $T$. aestivum ssp. vulgare, $T$. umbellulatum and $T$. variabile. These sources of resistance may be useful in bread wheat breeding. Several lines of diploid and tetraploid wheats could be used as additional discriminant hosts to separate pathotypes $\mathrm{Ha} 12$ and $\mathrm{Ha} 41$ of $H$. avenae.

Additional key words : Nematode, pathotype, wheat, resistance breeding, differential host.

\section{INTRODUCTION}

Le nématode à kystes des céréales, Heterodera avenae Woll., est un sérieux déprédateur des cultures de blé, d'orge et d'avoine dans de nombreux pays (MEAGHER, 1977 ; HOLDEMAN \& WATSON, 1977 ; RITTER, 1982). Parmi les méthodes capables de réduire l'incidence de ce ravageur, l'utilisation de variétés résistantes constitue une approche intéressante qui a motivé le développement d'importantes recherches au niveau de la sélection (COOK \& YORK, 1982). Celles-ci ont été entreprises en Suède, dès le début du siècle, par NiLSSON-EHLE (1920) qui signale pour la première fois la résistance de cultivars d'orge au développement d' $H$. avenae.

Ces recherches ont été intensifiées à partir de 1950 dans plusieurs pays d'Europe septentrionale, plus particulièrement pour l'orge et l'avoine chez lesquelles de nombreuses sources de résistance ont été caractérisées et sont maintenant couramment utilisées dans les programmes de sélection. Les travaux engagés ont d'ailleurs abouti à la création de cultivars à potentialités agronomiques satisfaisantes (ANDERSEN, 1980; COOK, 1974).

Chez le blé, les recherches sur la résistance à $H$. avenae ont été comparativement moins développées, bien que plusieurs cultivars résistants aient été identifiés chez Triticum aestivum $\mathrm{L}$. et $T$. durum Desf. en Inde (SIKORA et al., 1972 ; JAIN \& SEHGAL, 1979), en U.R.S.S. (ShIABOVA, 1979) ou en Australie (BROWN, 1982 ; O'BRIEN \& FISHER, 1974). En Europe et en Australie, 2 sources de résistance sont essentiellement utilisées dans les programmes de sélection. Il s'agit de T. aestivum cv. "Aus. 10894 " et ses descendants parmi lesquels le cultivar « Loros » dont la résistance est commandée par un système monogénique dominant situé sur le chromosome 2B (SLOOTMAKER et al., 1974) avec de possibles gènes modificateurs supplé- 
mentaires (COOK \& YORK, 1982 ; O'BRIEN et al., 1980) et de la variété «Festiguay » (FISHER J. M., comm. pers.).

La sélection de blés résistants à $H$. avenae utilise un nombre restreint de gènes dont l'efficacité est cependant incomplète à l'égard de pathotypes caractérisés notamment en Australie (ANDERSEN \& ANDERSEN, 1982 ; FISHER, 1982). Il est donc souhaitable de pouvoir disposer d'autres sources de résistance pour éviter le contournement éventuel par des pathotypes de virulence différente (ANDERSEN, 1980).

Diverses investigations chez des espèces voisines du blé ont permis de déceler de hauts niveaux de résistance, notamment chez $T$. variabile Eig. qui présente une efficacité totale en Australie (Brown, 1974). Ce résultat est en partie confirmé en France puisque 2 lignées de $T$. variabile expriment une résistance très forte, bien qu'incomplète, au développement du ravageur (DOSBA \& RivOAL, 1982). Ces auteurs ont également caractérisé un niveau quasi absolu de résistance chez plusieurs lignées de $T$. ventricosum (Ces.) Pass. $\&$ Gib. et de ses progéniteurs possibles du génome $\mathrm{M}^{\mathrm{v}}: T$. comosum (Sbith \& Sm.) Richt. et $T$. uniaristatum Richt.

L'analyse des lignées d'addition $T$. aestivum $\times T$. ventricosum a montré qu'une partie de la résistance est localisée sur le génome $\mathbf{M}^{\mathrm{v}}$ (DOSBA \& RIVOAL, 1981). Des travaux récents ont confirmé la localisation des gènes de résistance dont l'efficacité est liée à un effet de dosage chromosomique (RIVOAL et al., 1986). La résistance portée par le génome $M^{v}$ implique vraisemblablement un système génétique différent de celui qui a été identifié chez $T$. aestivum $\mathrm{cv}$. «Loros》 (SlOOTMAKER et al., 1974 ; O'BRIEN et al., 1980 ; Holm NiELSEN, 1982).

La présente publication fournit les résultats d'une large prospection réalisée de 1977 à 1984 pour identifier et évaluer l'efficacité d'éventuelles sources de résistance chez diverses espèces de Triticum.

\section{MATÉRIEL ET MÉTHODES}

La démarche méthodologique est identique à celle que nous avions adoptée pour caractériser les pathotypes d' $H$. avenae et la résistance de différentes lignées d'addition ( $T$. aestivum $\times T$. ventricosum) (RIVOAL, 1977 ; DOSBA \& RIVOAL, 1981).

\section{A. Matériel végétal}

88 lignées ou hybrides appartenant à 7 espèces de Triticum ont été confrontés aux pathotypes Fr1 et Fr4 d' $H$. avenae, respectivement désignés $\mathrm{Ha} 41$ et $\mathrm{Ha} 12$ par ANDERSEN \& ANDERSEN (1982). Ces 2 pathotypes couvrent le spectre de virulence qu'exprime le polymorphisme génétique de ce ravageur dans notre pays. Les lignées testées sont les suivantes :

Triticum monococcum (L.) Mk. $(2 \mathrm{n}=14$, génome A)

- ssp. beoticum (Boiss.) Mk. nos 828, 1038, 1039,

1040 ( $=T$. beoticum dans le texte, T.b. dans les figures de résultats)

- ssp. monococcum $\mathrm{n}^{\mathrm{os}} 157,830,835,7549,7550$,
$7552,7553,81187,81265(=T$. monococcum, T.m.)

Triticum urartu Tum $\left(2 \mathrm{n}=14\right.$, génome $\left.\mathrm{A}^{\mathrm{u}}\right) \mathrm{n}^{\mathrm{os}} 7796$, 7798 (= T.u.)

Triticum turgidum (L.) Thell. $(2 \mathrm{n}=28$, génomes $\mathrm{AB})$

- ssp. dicoccoides (Korn.) Thell. $\mathrm{n}^{\text {os }} 4$, 829, 7431, 7432 (= T. dicoccoides, T.ds)

- spp. dicoccum (Schrank) Thell. $\mathrm{n}^{\mathrm{os}} 8,22,31 \mathrm{~A}, 64$, 487, 81098, «Khapli », «Emmer $417 »(=T$. dicoccum, T.dm)

- ssp. carthlicum (Nevski) Mk. nos 305, $360(=T$. carthlicum, T.c.)

- ssp. turgidum L. conv. turgidum $\mathrm{n}^{\mathrm{os}} 226,227,228$, $229,231,488 \mathrm{~A}, 488 \mathrm{~B}, 7054,7055,7056,7057$, $7059,7062,7090 \mathrm{~B}_{1}, 7351,7427,7452(=$ T. turgidum, T.t.)

conv. durum (Desf.) Mk. $\mathrm{n}^{\text {os }} 487,75180,7654=$ $(1708 \times$ «Skopje $»), 7655=(«$ Oviatuc » $\times$ «Skopje »), CF 84, I 32, « Sansome 201p» $(=T$. durum, T.d.)

conv. aethiopicum Jakubz. $\mathrm{n}^{\text {os }} 1 \mathrm{~A}, 403(=T$. aethiopicum, T.am)

Triticum timopheevi Zhuk. ( $2 \mathrm{n}=28$, génomes $\mathrm{AG})$

- ssp. araraticum (Jakubz.) Mk. $\mathrm{n}^{\text {os }} 77108,77109$, $77110,77111,77112(=T$. araraticum, T.a.)

- ssp. timopheevi $\mathrm{n}^{\mathrm{os}} 805,6906 \mathrm{~B}, 7202 \mathrm{~B}(=$ T. timopheevi, T.ti)

Triticum aestivum L. $(2 \mathrm{n}=42$, génomes $\mathrm{ABD})$

- ssp. spelta (L.) Thell. : «Altgold», H 9651, « Rouquin » $(=T$. spelta, T.s. $)$

- ssp. vulgare (Vill.) Mk. : « Hardi », « Moisson », "Roazon », VPM $=\{($ Aegilops ventricosa $\times$ Triticum persicum) $\times$ « Marne $\left.{ }^{3} »\right\}$ lignées 2 et 7 , "Chinese Spring », \{ « Loros » $\times$ F1 (« Capitole » $\times$ « Roazon »)\} lignées 29-1 et 29-4, 8203, $8204,8205,8206,8207,8208$ (= T. vulgare, T.v.)

- lignée d'addition (blé- $T$. ventricosum) $\mathrm{m} 36(2 \mathrm{n}=$ 44, génomes $\mathrm{ABD}+1$ paire chromosomique du génome $\mathrm{M}^{\mathrm{v}}$ ) (= T.v.)

Hybrides F1 = (Triticum aestivum cv. "Chinese Spring " $\times$ Triticum variabile 1$)$ et $(T$. aestivum $\mathrm{cv}$. "Chinese Spring" $\times T$. variabile 6) $(2 \mathrm{n}=35$, génomes $\left.\mathrm{ABDUS}{ }^{\vee}\right)$. Ces hybrides ont été produits sans avoir recours à la culture in vitro d'embryons immatures ; le blé tendre cv. "Chinese Spring » a été le parent femelle (= T.v.).

Triticum umbellulatum (Zhuk.) Bowden $(2 \mathrm{n}=14$, génome U) $\mathrm{n}^{\mathrm{os}} 1,3,5$ (= T.um).

Triticum variabile Eig $(2 \mathrm{n}=28$, génomes US $) \mathrm{n}^{\mathrm{os}} 1,6$ (= T.ve).

Les blés tendres $T$. aestivum cv. « Hardi » et cv. «Moisson » ont été les témoins hôtes dans les différents tests.

\section{B. Evaluation de la résistance}

Elle est réalisée en conditions extérieures à l'aide de 2 techniques précédemment décrites (RIvOAL et al., 1978). Un $1^{\text {er }}$ tri des lignées est effectué sur des cultures en drains dans le sol naturellement infesté. Le niveau de résistance est évalué ensuite après culture, en bouteilles, dans un compost artificiellement contaminé par l'apport d'un nombre connu de kystes pleins, nouvelle- 
ment formés, issus d'élevages de l'année. Les tests sont menés avec 5 répétitions dans les expérimentations «drains » et 4 dans les expérimentations « bouteilles ». Les populations de nématodes utilisées sont celles de Villasavary (Aude) pour Ha4l et de Nuisement-sur-Coole (Marne) pour Hal2.

Les semis, à raison d'un caryopse germé par drain ou par bouteille, interviennent dans le courant du mois de novembre. Les observations sont effectuées en juin pour les drains, en septembre pour les bouteilles. Dans le $1^{\text {er }}$ cas, on procède à une estimation ou à un dénombrement des femelles blanches du parasite sous le microscope stéréoscopique, après lavage des racines. Dans le $2^{\mathrm{e}}$ cas, les kystes sont extraits par élutriation et centrifugation, puis recensés sous le même dispositif optique. Les résultats sont fournis en nombres moyens de femelles blanches ou de kystes par plante.

Il est facile de caractériser une résistance complète qui se traduit par l'absence totale de femelle. On définit plus difficilement des résistances partielles d'autant que l'expression du caractère hôte des témoins peut varier. Le nombre des femelles est en effet l'expression phénotypique d'une interaction entre les caractéristiques de la plante et les conditions expérimentales. Des lignées peuvent présenter une qualité d'hôte intermédiaire entre les témoins multiplicateurs et la résistance totale. On a donc caractérisé les lignées par le nombre moyen de femelles ou de kystes formés par rapport aux témoins selon un classement continu plutôt que d'établir un seuil arbitraire de résistance comme l'a fait BROWN (1969).

\section{RÉSULTATS}

Les données des investigations en sol naturellement infesté sont fournies dans les figures 1,2 et 3 . Les résultats d'évaluation du niveau de résistance en sol artificiellement infesté sont donnés dans la figure 4.

\section{A. Blés diploïdes}

Les 4 lignées de $T$. beoticum se révèlent hôtes multiplicateurs des 2 pathotypes d' $H$. avenae (fig. 1, A et C). Par contre une variabilité dans la capacité à multiplier le parasite est observée chez $T$. urartu dont les 2 lignées 7796 et 7798 testées respectivement en sol naturellement et artificiellement infesté sont des hôtes efficaces pour Ha12 et médiocres pour Ha41 (fig. 1, B) et (fig. 4, A). Des observations similaires sont faites pour $T$. monococcum. En 1978 et en 1982, la lignée 830 présente un niveau intermédiaire de résistance qui s'exprime d'ailleurs mieux dans le cas de Ha12 que dans celui de $\mathrm{Ha} 4 \mathrm{l}$ (fig. 1, A et C). La faible capacité de cette lignée à multiplier ces 2 écotypes s'observe à nouveau en 1979, avec des conditions artificielles d'infestation (fig. 4, A).

En 1982, on décèle à nouveau un haut niveau de résistance chez plusieurs lignées de $T$. monococcum telles que 7549,7552 et 7553 . La lignée 7552 s'oppose totalement au développement des 2 pathotypes (fig. 1, C) ; la lignée 157 se comporte également en hôte médiocre plus particulièrement dans le cas de Ha41.

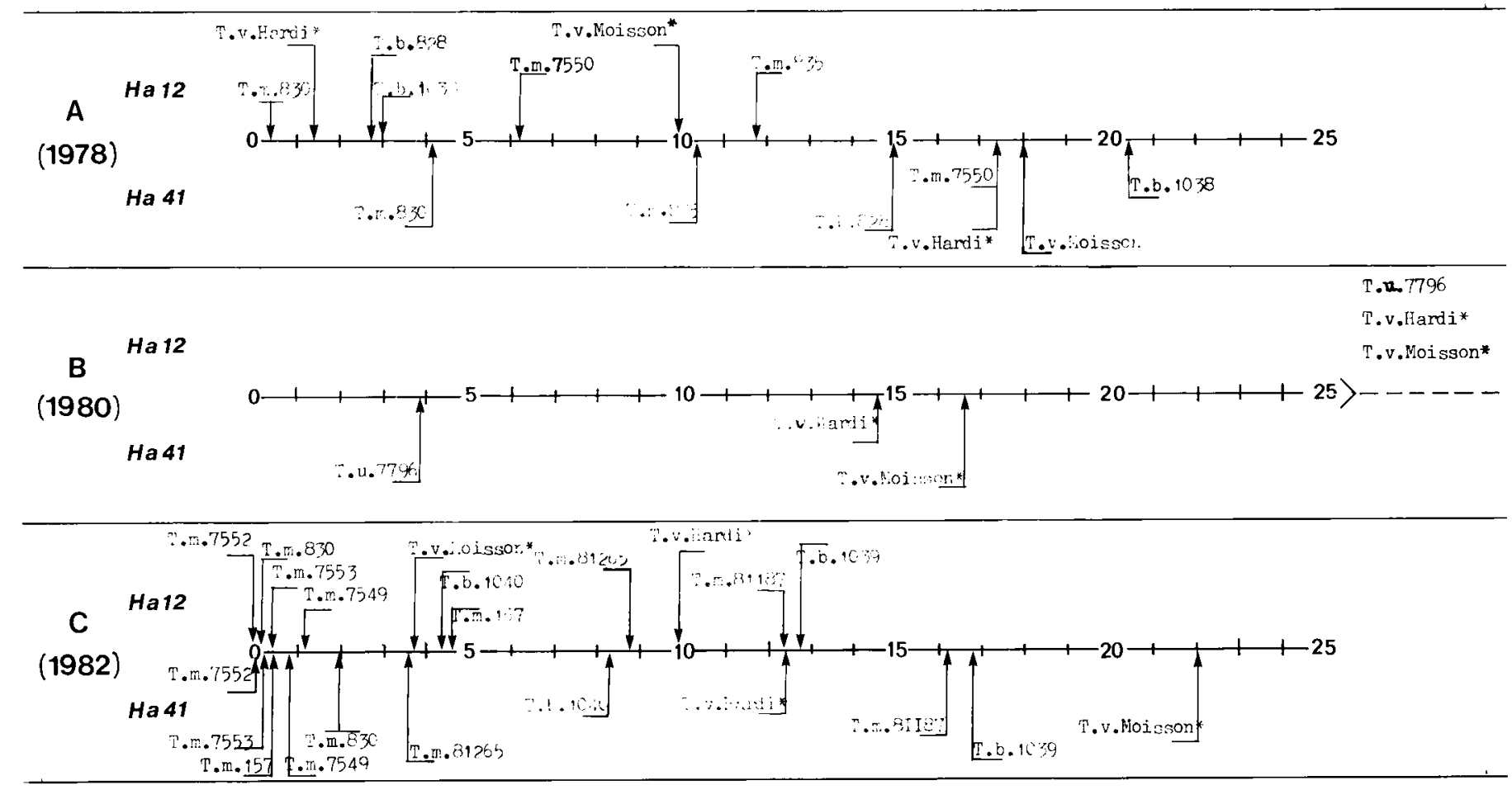

Figure 1

Classement de blés diplö̈des en fonction du nombre moyen de femelles blanches d'H. avenae ; résultats de tests menés en sol naturellement infesté ( $^{*}=$ témoins hôtes).
Classification of diploid wheats according to average number of white females of $\mathrm{H}$. avenae ; results of experiments carried out in naturally infested soil $\left(^{*}=\right.$ control hosts).

T.b. = T. monococcum ssp. beoticum $;$ T.m. = T. monococcum ssp. monococcum ; T.u. $=$ T. urartu ; T.v. $=$ T. aestivum ssp. vulgare. 


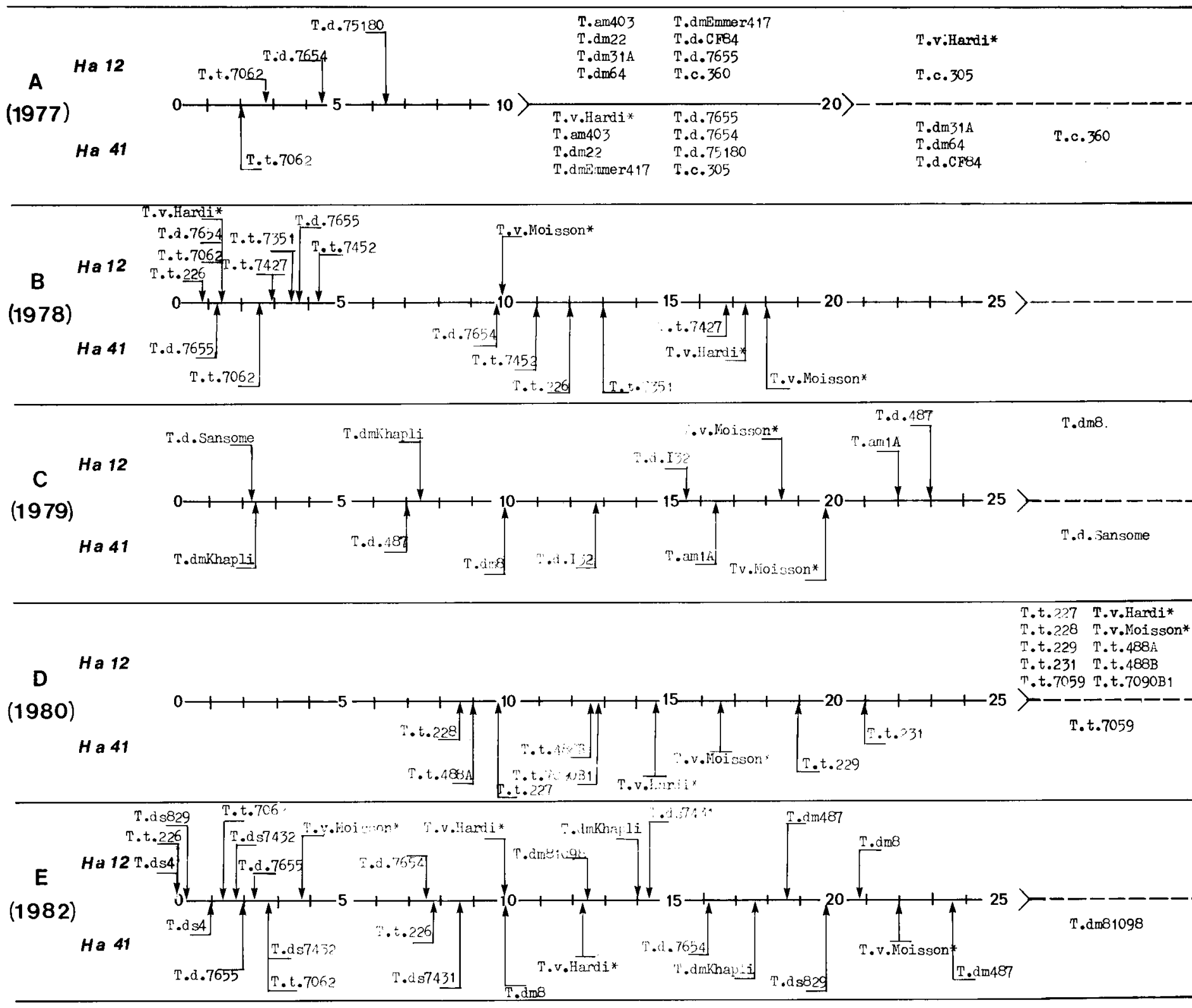

Figure 2

Classement de blés tétraploïdes (T. turgidum) en fonction du nombre moyen de femelles blanches d'H. avenae ; résultats de tests menés en sol naturellement infesté $\left(^{*}=\right.$ témoins hôtes).

\section{B. Blés tétraploïdes}

T. carthlicum (2 lignées) et $T$. aethiopicum (2 lignées) ne fournissent aucun génotype résistant. Parmi les 8 lignées de $T$. dicoccum, seule la lignée «Khapli » s'oppose nettement à la multiplication de Ha41 (fig. 2, C). Chez T. durum, une opposition au développement d' $H$. avenae est constatée dans la lignée 7655 aussi bien en 1978 qu'en 1982 (fig. 2, B et E). Quelle que soit l'année du test, la résistance de cette lignée est plus forte à l'encontre de Ha4l que de Ha12 ; cette différence d'efficacité est observée même en conditions d'infestation contrôlées (fig. 4, A). En 1979, la lignée « Sansome 201p » se comporte en hôte sélectif à l'égard des 2 pathotypes en multipliant nettement plus Ha41 que Ha12. T. turgidum lignée 7062 présente éga-
Classification of tetraploid wheats (T. turgidum) according to average number of white females of $\mathrm{H}$. avenae; results of experiments carried out in naturally infested soil $1^{*}=$ control hosts).

$T . d s=s s p$. dicoccoides $; T . d m=s s p$. dicoccum ; T.c. $=s s p$. carthlicum ; T.t. = ssp. turgidum conv. turgidum; T.d. $=s s p$. turgidum conv. durum; T.am. = ssp. turgidum conv. aethiopicum $; T . v .=\mathrm{T}$. aestivum ssp. vulgare.

lement une forte capacité à empêcher la multiplication des 2 pathotypes $\mathrm{Ha} 12$ et $\mathrm{Ha} 41$ (fig. 2, A, B et E). Cette résistance se révèle cependant moins efficace en conditions d'infestation artificielle (fig. 4, A). Chez T. dicoccoides, la lignée 4 présente le meilleur niveau de résistance au développement des 2 pathotypes (fig. 2, E). La lignée 829 s'oppose au développement de $\mathrm{Ha} 12$ et la lignée 7432 est un hôte médiocre à la fois pour $\mathrm{Ha} 12$ et $\mathrm{Ha} 41$.

Aucune des 3 lignées de $T$. timopheevi testées en 1977 ne s'oppose à la multiplication d' $H$. avenae (fig. 3, A). Par contre les 3 lignées 77109, 77111 et 77112 de $T$. araraticum présentent un nombre très faible et parfois nul de femelles des 2 pathotypes Ha12 et Ha41, en 1982 (fig. 3, B). 


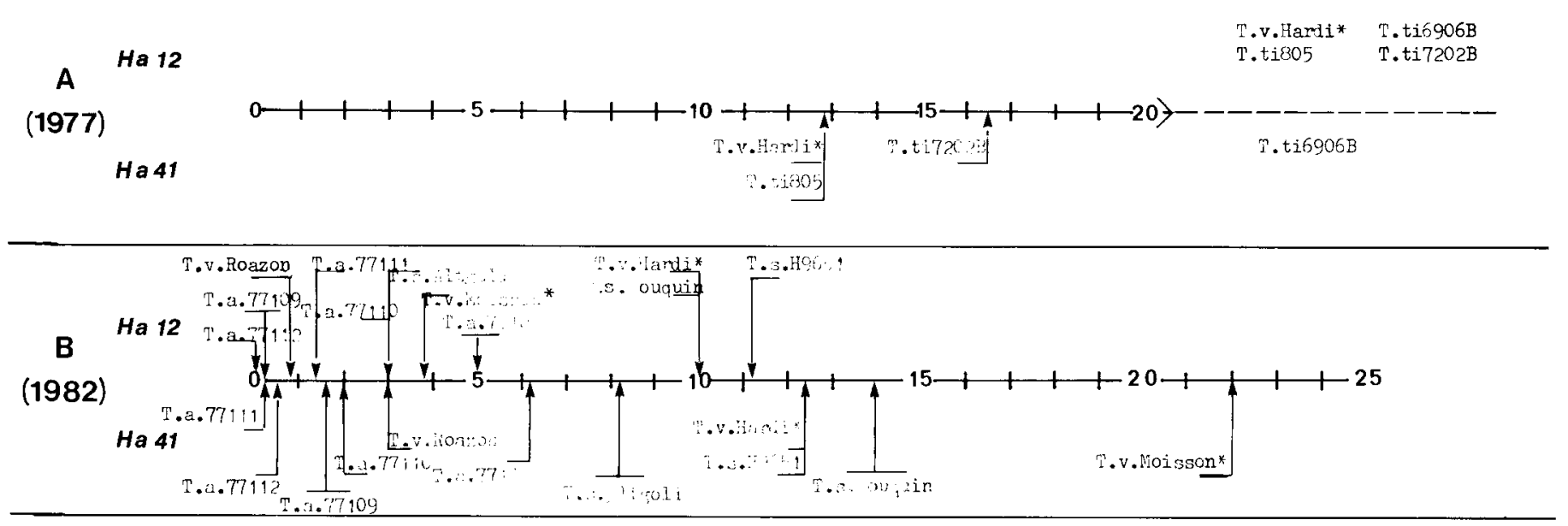

Figure 3

Classement de blés tétraplö̈des (T. timopheevi) et hexaplö̈des (T. aestivum) en fonction du nombre moyen de femelles blanches $d ' \mathrm{H}$. avenae ; résultats de tests menés en sol naturellement infesté $1^{*}=$ témoins hôtes $)$.

\section{Blés hexaploïdes}

Un niveau de résistance intermédiaire à l'encontre de Ha41 est enregistré chez VPM, lignée 2 (fig. 4, A). Le blé Roazon qui en est issu présente également une qualité d'hôte médiocre à l'égard des deux pathotypes (fig. 3, B). La lignée d'addition m36 confirme également un niveau de résistance intermédiaire très intéressant à l'encontre du pathotype Ha12 (fig. 4, B). Aucune des lignées 8203 à 8208 ne s'oppose efficacement à la multiplication du pathotype Ha12. Il n'y a pas non plus de résistance chez les 3 lignées de T. spelta analysées (fig. 3, B).

\section{T. umbellulatum et $T$. variabile (fig. $4, \mathrm{C}$ )}

Les 3 lignées de $T$. umbellulatum testées en conditions artificielles d'infestation en 1984 présentent un haut niveau de résistance à l'encontre de Ha12 (fig. 4, B). Vis-à-vis du même pathotype, les 2 lignées de $T$. variabile ont une résistance quasi totale. On la retrouve chez l'hybride («Chinese Spring» $\times T$. variabile $\left.\mathrm{n}^{\circ} 1\right)$. L'hybride obtenu à partir de la lignée $n^{\circ} 6$ possède une résistance moins efficace, supérieure cependant à celle des lignées de $T$. umbellulatum.

\section{DISCUSSION-CONCLUSION}

La phase préliminaire de prospection réalisée en sol naturellement infesté est une approche de la mesure du niveau de résistance. Elle est menée dans des conditions expérimentales insuffisamment contrôlées au niveau de l'infestation et les effectifs en femelles blanches sont en outre sous-estimés du fait de la perte d'un nombre inconnu d'individus au cours du lavage des racines. Néanmoins cette caractérisation s'est révélée fiable puisque, testés pendant 2 ou 3 années, $T$. monococcum 830, T. turgidum 7062 et $T$. durum
Classification of tetraploid (T. timopheevi) and hexaploid (T. aestivum) wheats, according to average number of white females of $\mathrm{H}$. avenae ; results of experiments carried out in naturally infested soil ${ }^{*}=$ control hosts).

T.a. $=s s p$. araraticum $;$ T.ti $=s s p$. timopheevi $;$ T.s. $=\mathrm{T}$. spelta $;$ $T . v .=\mathrm{T}$. aestivum ssp. vulgare.

7655 présentent une qualité constante d'hôte médiocre à l'égard des 2 pathotypes d' $H$. avenae. La vérification de ces résultats par le test en sol artificiellement infesté qui assure une infestation homogène et une plus forte multiplication du nématode est cependant nécessaire. Elle permet de mieux évaluer le niveau de résistance des lignées repérées. Ainsi il s'avère que T. monococcum 830 possède une résistance moins efficace que celle qui avait été précédemment décelée à l'égard des 2 pathotypes.

Cette étude a permis de mettre en évidence différents niveaux de résistance. Les plus élevés sont observés chez $T$. aestivum lignées 29.1 et 29.4 issues de «Loros », qui sont déjà utilisées dans nos programmes de sélection. La résistance quasi totale de la lignée $\mathrm{n}^{\circ} 1$ de $T$. variabile à l'encontre de Ha12 confirme les résultats de DosBA \& RivoAL (1981). Ce génotype a la particularité d'être résistant à Meloidogyne naasi Franklin, nématode à galle des racines de céréales (PERSON-DEDRYVER \& JAHIER, 1985). Le haut niveau de résistance de l'hybride F1 ( Chinese Spring » $\times T$. variabile $n^{\circ}$ 1) augmente l'intérêt que l'on doit également porter à cette espèce dans les programmes de sélection. Il en est de même pour les 3 lignées de $T$. umbellulatum qui présentent aussi une résistance efficace à l'encontre de $M$. naasi (PERSONDEDRYVER \& JAHIER, 1985). Un nombre élevé de lignées à résistance incomplète est en outre observé chez $T$. monococcum ( ${ }^{\text {os }} 7549,7552$ et 7753), $T$. durum $\mathrm{n}^{\circ} 7655, T$. turgidum $\mathrm{n}^{\circ} 7062$ et chez $T$. araraticum ( ${ }^{\text {os }} 77109$ à 77112 ).

Cette prospection contribue à élargir nos connaissances sur les hôtes potentiels d' $H$. avenae et apporte des informations complémentaires à la synthèse établie par GOODEY et al. (1965) et aux résultats obtenus en Australie par BROWN \& MEAGHER (1970). Elle précise les relations entre la diversité génétique de lignées de triticinées et la virulence d'H. avenae en France. En effet, à la différence de $T$. aestivum, certains blés 


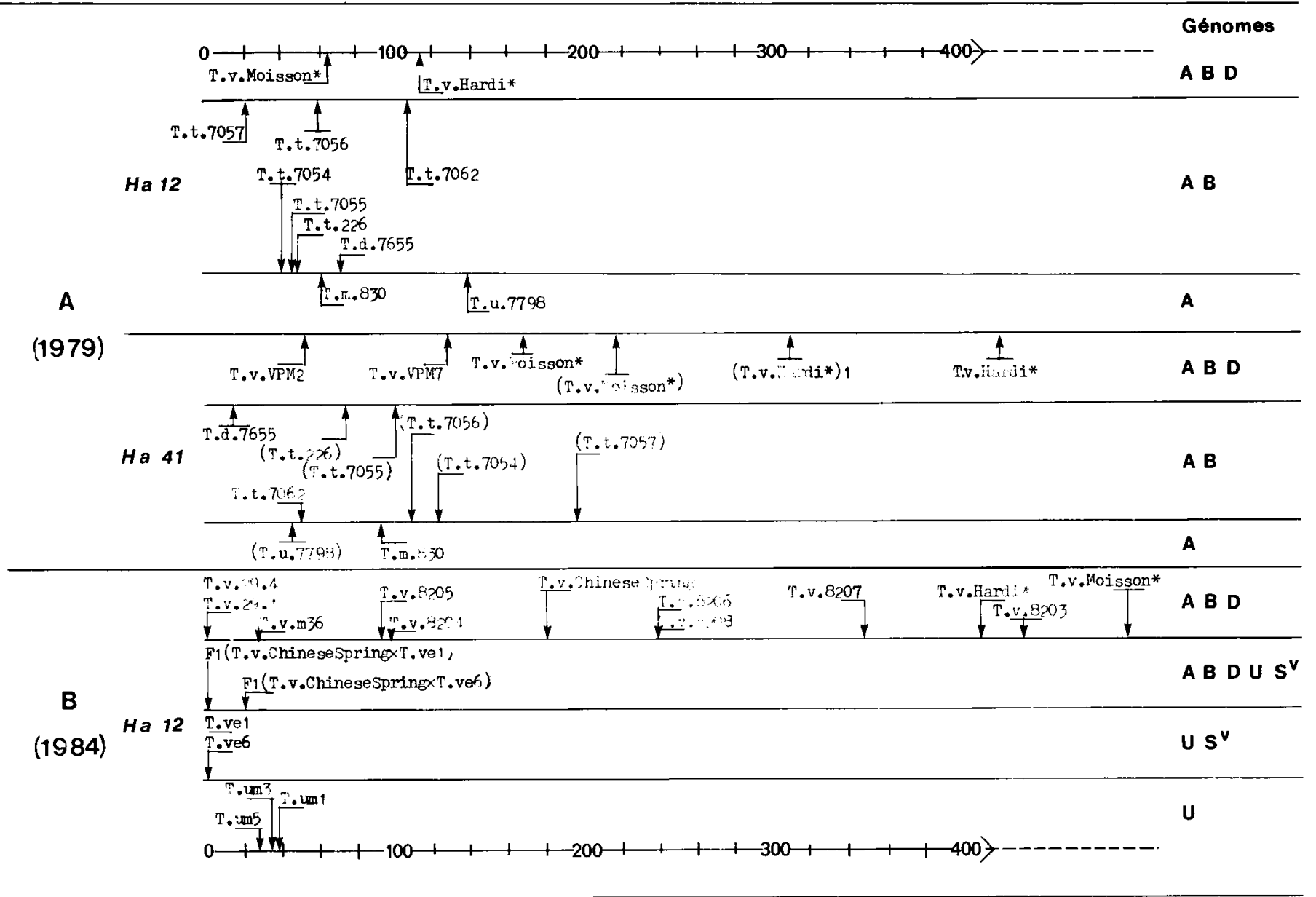

Figure 4

Niveaux de résistance de diverses espèces de Triticum selon le nombre moyen de kystes gravides d'H. avenae : résultats de tests menés en sol artificiellement infesté $\left(^{*}=\right.$ témoins hôtes).

diploïdes ( $T$. beoticum 828, T. urartu 7796, T. monococcum 157) et tétraploïdes ( $T$. durum «Sansome $201 \mathrm{p}$ » et $T$. dicoccoides 829 ) présentent une qualité d'hôte différente pour les 2 pathotypes $\mathrm{Ha} 12$ et $\mathrm{Ha} 41$. Ils pourraient constituer des hôtes différentiels pour séparer les pathotypes d' $H$. avenae en Europe septentrionale.

Ces résultats indiquent également que la résistance à la multiplication d' $H$. avenae existe chez plusieurs espèces du genre Triticum. Il convient d'évaluer l'efficacité de ces sources à l'encontre du plus grand nombre de pathotypes, avant de choisir les géniteurs. L'étude de la localisation des gènes de résistance et de leur mécanisme d'action devrait compléter ces observations afin de s'assurer de la réelle diversité des sources de résistance repérées. Il nous paraît en effet indispensable de préparer dès maintenant une alternative à l'utilisation du géniteur «Loros » dans les programmes d'amélioration du blé en France.
Resistance levels of several species of Triticum in relation to average number of gravid cysts of $\mathrm{H}$. avenae : results of experiments carried out in artificially infested soil $\left(^{*}=\right.$ control hosts).

T.m. $=\mathrm{T}$. monococcum $;$ T.u. $=\mathrm{T}$. urartu $;$ T.t. $=\mathrm{T}$. turgidum $;$ T.d. $=\mathrm{T}$. durum $;$ T.um $=$ umbellulatum $; T . v e=\mathrm{T}$. variabile $T . v .=\mathrm{T}$. aestivum ssp. vulgare.

Les différentes espèces du genre Triticum offrent donc de réelles possibilités pour diversifier les sources de résistance. Leur utilisation pour améliorer la résistance du blé aux nématodes ( $H$. avenae et $M$. naasi) ainsi qu'aux maladies fongiques est engagée car on dispose de techniques de manipulation chromosomique qui permettent l'introgression dans le blé, de gènes appartenant à d'autres triticinées (LAW, 1981).

Reçu le 5 février 1986

Accepté le 24 avril 1986.

\section{REMERCIEMENTS}

Les auteurs remercient J, M. Fisher, Department of Plant Pathology, Waite Agricultural Research Institute, University of Adelaide, Glen Osmond (South Australia), pour la fourniture des lignées 8203 à 8208 de T. aestivum. 


\section{RÉFÉRENCES BIBLIOGRAPHIQUES}

Andersen S., 1980. The breeding and use of varieties resistant to Heterodera avenae. EPPO Bull., 10, 303-310.

Andersen S., Andersen K., 1982. Suggestions for determination and terminology of pathotypes and genes for resistance in cyst-forming nematodes, especially Heterodera avenae. EPPO Bull., 12, 379-386.

Brown J. A. M., 1974. Test tube reproduction of Heterodera avenae on resistance and susceptible wheats. Nematologica, 20, 192203.

Brown R. H., 1969. The occurence of biotypes of the cereal cyst nematode (Heterodera avenae Woll.) in Victoria. Austr. J. exp. Agr. anim. Husb., 9, 453-456.

Brown R. H., 1982. Studies on the Australian pathotype of Heterodera avenae. EPPO Bull., 12, 413-416.

Brown R. H., Meagher J. W., 1970. Resistance in cereals to the cyst nematode (Heterodera avenae) in Victoria. Aust. J. exp. Agr. anim. Husb., 10, 360-365.

Cook R., 1974. Nature and inheritance of nematode resistance in cereals. J. Nematol., 6, 165-174.

Cook R., York P. A., 1982. Resistance of cereals to Heterodera avenae : methods of investigation, sources and inheritance of resistance. EPPO Bull., 12, 423-434.

Dosba F., Rivoal R., 1981. Les lignées d'addition blé-Aegilops ventricosa Tausch. II. Etude de leur comportement et.de celui de leurs progéniteurs vis-à-vis d'Heterodera avenae Woll. Agronomie, 1 (7), 559-564.

Dosba F., Rivoal R., 1982. Estimation des niveaux de résistance au développement d'Heterodera avenae chez les triticinées. EPPO Bull., 12, 451-456.

Fisher J. M., 1982. Problems with the use of resistance in wheat to the Australian pathotype of Heterodera avenae. EPPO Bull., 12, 417-421.

Goodey J. B., Franklin M. T., Hooper D. J., 1965. The nematode parasites of plants catalogued under their hosts. Commonw. Agric. Bureaux, Farnham Royal, Bucks, England, 214 p.

Holdeman Q. L., Watson T. R., 1977. The oat cyst nematode Heterodera avenae Wollenweber, 1924: a root parasite of cereal crops and other grasses. St. Calif. : Dep. Food \& Agric., 82 p.

Holm Nielsen C., 1982. Heredity of Heterodera avenae resistance originating from two barley cultivars and one spring wheat cultivar. EPPO Bull., 12, 457-461.

Jain R. K., Sehgal S. P., 1979. Note on the reaction of some wheat varieties to the cereal cyst nematode. Ind. J. agric. Sci., 46, 213-214.
Law C. N., 1981. Chromosome manipulation in wheat. Chromosomes today, 7, 194-295.

Meagher J. W., 1977. World dissemination of the cereal-cyst nematode (Heterodera avenae) and its potential as a pathogen of wheat. J. Nematol., 9, 9-15.

Nilsson-Ehle H., 1920. Über Resistenz gegen Heterodera schachtii bei gewissen Gerstensorten, ihre Vererbungsweise und Bedeutung für die Praxis. Hereditas, 1, 1-34.

O'Brien P. C., Fisher J. M., 1974. Resistance within wheat, barley and oat cultivars to Heterodera avenae in South Australia. Aust. J. exp. Agric. anim. Husb., 14, 399-404.

O'Brien P. C., Fisher J. M., Rathjen A. J., 1980. Inheritance of resistance in two wheat cultivars to an Australian population of Heterodera avenae. Nematologica, 26, 69-74.

Person-Dedryver F., Jahier J., 1985. Les céréales à paille hôtes de Meloidogyne naasi Franklin. III. Recherches de sources de résistance parmi les espèces voisines du blé tendre. Agronomie, 5 (7), 573-578.

Ritter M., 1982. Importance des nématodes à kystes des céréales. Bull. OEPP, 12, 307-316.

Rivoal R., 1977. Identification des races biologiques du nématode à kystes des céréales, Heterodera avenae Woll. en France. Ann. Zool. Ecol. anim., 9, 261-272.

Rivoal R., Person F., Caubel G., Scotto La Massese C., 1978. Méthodes d'évaluation de la résistance des céréales au développement des nématodes : Ditylenchus dipsaci, Heterodera avenae et Pratylenchus spp.. Ann. Amélior. Plantes, 28, 371-394.

Rivoal R., Dosba F., Jahier J., Pierre J. S., 1986. Les lignées d'addition blé-Aegilops ventricosa Tausch. VI : Etude de la localisation chromosomique de la résistance à l'égard d'Heterodera avenae Woll.. Agronomie, 6 (2), 143-148.

Shiabova T. N., 1979. (Study of the resistance of cereal crops to Heterodera avenae). In : Printsipy $i$ metody izucheniya vzaimootnoshenii mezhdu paraziticheskimi nematodami i rasteniyami. Tartu, USSR ; Akademiya Nauk Estonskoï SSR, 83-89.

Sikora R. A., Koshy P. K., Malek R. B., 1972. Evaluation of wheat selections for resistance to the cereal cyst nematode. Ind. J. Nematol., 2, 81-82.

Slootmaker L. A. J., Lange W., Jochemsen G., Schepers J., 1974. Monosomic analysis in bread wheat of resistance to cereal root eelworm. Euphytica, 23, 497-503. 